\title{
Observation of Spatiotemporal Self-Focusing of Spin Waves in Magnetic Films
}

\author{
M. Bauer, O. Büttner, S. O. Demokritov,* and B. Hillebrands \\ Fachbereich Physik and Forschungs- und Entwicklungsschwerpunkt Materialwissenschaften, Universität Kaiserslautern, \\ Erwin-Schrödinger-Strasse 46, 67663 Kaiserslautern, Germany \\ V. Grimalsky and Yu. Rapoport \\ T. Shevchenko Kiev State University, 252601 Kiev, Ukraine
}

A. N. Slavin

Department of Physics, Oakland University, Rochester, Michigan 48309-4401

(Received 15 July 1998)

\begin{abstract}
The first observation of spatiotemporal self-focusing of spin waves is reported. The experimental results are obtained for dipolar spin waves in yttrium-iron-garnet films by means of a newly developed space- and time-resolved Brillouin light scattering technique. They demonstrate self-focusing of a moving wave pulse in two spatial dimensions, and formation of localized two-dimensional wave packets, the collapse of which is stopped by dissipation. The experimental results are in good qualitative agreement with numerical simulations. [S0031-9007(98)07425-0]

PACS numbers: 75.30.Ds, 76.50.+g, 78.35.+c
\end{abstract}

Nonlinear self-focusing of wave beams and pulses is an important phenomenon in physics because it provides a mechanism for localization of wave energy in a small spatial region [1]. In a simple model based on the nonlinear Schrödinger equation with two or three spatial dimensions, self-focusing for certain initial conditions leads to the collapse of the initial wave packet, when the packet amplitude becomes infinite in a finite time [2]. In real physical experiments singularity is, of course, avoided, and the process of collapse is stopped by saturation of nonlinearity and/or by dissipation. The effects of strong self-focusing and of wave collapse have been observed thus far for light waves in nonlinear optics [3], and for nonlinear Langmuir waves in a plasma [4]. The possibility of "light bullets," i.e., stable optical wave pulses strongly localized in space and time by self-focusing, which is stabilized by saturation of nonlinearity at high wave amplitudes, has been suggested by Silberberg [5]. There exists, however, no experimental evidence for this effect in optics, likely because, in optical fibers, diffraction is much stronger than dispersion, and, therefore, both effects cannot be observed simultaneously.

In this Letter we report the first experimental observation of spatiotemporal self-focusing of dipolar spin waves and formation of strongly localized two-dimensional wave packets (spin wave bullets) propagating in yttrium-irongarnet (YIG) films. Here the diffraction to dispersion ratio is much smaller than in optical fibers, which makes it possible to observe a simultaneous self-focusing of a propagating spin wave packet along both in-plane directions. The "spin wave bullets" in our experiments are formed as a result of a spatiotemporal self-focusing, similar to the self-focusing effect described in Ref. [5], as our two-dimensional input spin wave packets are self-focused along both in-plane directions ( $y$ and $z$ ), while propagat- ing along one of them $(z)$. We note, however, that selffocusing of dipolar spin waves in YIG films is stabilized by dissipation, rather than by saturation of nonlinearity, as was suggested for light bullets in Ref. [5].

It is well-known [6,7], that, in the absence of dissipation, two-dimensional self-focusing of an input wave packet leads to a packet collapse only if the packet amplitude is sufficiently large for nonlinearity to overcome the effects of diffraction and dispersion. Thus, the twodimensional collapse is critical; i.e., it has an amplitude threshold even in a dissipationless medium.

In the presence of dissipation, the amplitude of a selffocusing wave packet decreases exponentially with time, and with the decrease of amplitude the focusing effect of nonlinearity also decreases. This leads to a collapse stabilization and subsequent defocusing of the packet when its amplitude becomes smaller than the collapse threshold [for details, see Eqs. (40)-(47) in Ref. [6] ].

The influence of dissipation on spin wave packets in YIG films is much stronger than the similar dissipative effect on light wave pulses in optical fibers [8]. Thus, the two-dimensional collapse of dipolar spin waves is rapidly stabilized by dissipation, and a quasistable self-focused spin wave packet-a spin wave bullet-is formed. Such a "bullet" propagates for a certain distance without changing its spatial sizes (but constantly losing energy due to dissipation). Then, at some point it starts to diverge in space, when its amplitude is not anymore sufficient for self-focusing. We stress that a spin wave bullet is not a stable two-dimensional envelope soliton, but a quasistable self-focusing wave packet, collapse of which has been stopped by dissipation.

The evolution of a spectrally narrow two-dimensional ( $y$ and $z$ ) spin wave packet, propagating along the $z$ direction in a magnetic film is usually described in the framework 
of a $(2+1)$-dimensional nonlinear Schrödinger (NLS) equation with dissipation (see [9] and references therein)

$$
\begin{aligned}
i\left(\frac{\partial U}{\partial t}+V_{g} \frac{\partial U}{\partial z}\right)+\frac{1}{2} D \frac{\partial^{2} U}{\partial z^{2}}+ & \\
S \frac{\partial^{2} U}{\partial y^{2}}-N|U|^{2} U & =-i \omega_{r} U,
\end{aligned}
$$

where $U$ is the dimensionless amplitude of the wave packet envelope $\left[U^{2}=m^{2} / 2 M_{0}^{2}=(1 / 2) \tan ^{2} \chi\right.$, where $m$ and $M_{0}$ are the amplitudes of the variable and saturation magnetizations, and $\chi$ is the magnetization precession angle], $V_{g}=\partial \omega / \partial k_{z}$ is the group velocity, $D=\partial^{2} \omega / \partial k_{z}^{2}$ and $S=\partial \omega / \partial k_{y}^{2}$ are the dispersion and diffraction coefficients, $N=\partial \omega / \partial|U|^{2}$ is the nonlinear coefficient, $\omega_{r}=$ $\gamma \Delta H$ is the dissipation parameter proportional to the ferromagnetic resonance (FMR) half-linewidth $\Delta H, \gamma / 2 \pi=$ $2.8 \mathrm{MHz} / \mathrm{Oe}$ is the gyromagnetic ratio, and $\omega\left(k_{y}, k_{z},|U|^{2}\right)$ is the nonlinear dispersion law for the spin waves propagating in the film.

Dipolar backward volume magnetostatic waves (BVMSW), propagating along the bias magnetic field in a tangentially magnetized YIG film, have been chosen for our experiments. The nonlinear coefficient $N$ for BVMSW modes is negative, while both the dispersion $(D)$ and the diffraction $(S)$ coefficients are positive [9]. Thus, plane BVMSW modes fulfill the Lighthill criterion [10] for modulational instability in both in-plane directions $(S N<0$ and $D N<0)$, and the effects of self-modulation (leading to the formation of temporal envelope solitons) and self-focusing (leading to the formation of stationary spatial solitons) are allowed for these waves. Both of these effects have been separately observed in YIG films earlier [11-14].

The aim of our present work is to study experimentally the evolution of propagating BVMSW pulse beams (or two-dimensional wave packets) that are simultaneously affected by nonlinearity, dispersion, diffraction, and dissipation. They are, therefore, susceptible to nonstationary spatiotemporal self-focusing and collapse [5].

In our experiments we used a large $\left(1.8 \times 2.6 \mathrm{~cm}^{2}\right)$ sample of a high-quality epitaxial YIG film [thickness $d=7 \mu \mathrm{m}$, FMR linewidth (at $8 \mathrm{GHz}) 2 \Delta H=0.6 \mathrm{Oe}$, saturation magnetization $4 \pi M_{0}=1750 \mathrm{G}$ ] magnetized to saturation by a tangential bias magnetic field of $H=2098$ Oe. A standard delay-line structure $[9,12]$ with two short-circuited microstrip antennas (length $l_{a}=2.5 \mathrm{~mm}$, width $W=50 \mu \mathrm{m}$ ) oriented along the $y$ axis, and separated by the distance $l=8 \mathrm{~mm}$ along the $z$ axis, was used for excitation and detection of the BVMSW packets. The carrier frequency of the input BVMSW packet was chosen at $f_{c}=7.97 \mathrm{GHz}, 30 \mathrm{MHz}$ lower than the upper boundary of the BVMSW spectrum, to allow propagation of the input pulses of duration $T>28 \mathrm{~ns}$ without significant distortions to their frequency spectra. The carrier wave number of the BVMSW packet at this frequency point was estimated to be $k_{z 0}=50 \mathrm{~cm}^{-1}$.
The coefficients of the NLS Eq. (1), calculated for this working point using the expressions given in [9], are $V_{g}=-4.0 \times 10^{6} \mathrm{~cm} / \mathrm{s}, \quad D=1.6 \times 10^{3} \mathrm{~cm}^{2} / \mathrm{s}, \quad S=$ $1.1 \times 10^{5} \mathrm{~cm}^{2} / \mathrm{s}, \quad N=-1.1 \times 10^{10} \mathrm{~s}^{-1}, \quad$ and $\omega_{r}=$ $\gamma \Delta H=5.2 \times 10^{6} \mathrm{~s}^{-1}$. The experimentally measured group velocity of the packet in the linear regime, $V_{g}=-(4.0 \pm 0.1) \times 10^{6} \mathrm{~cm} / \mathrm{s}$, was in good agreement with the theoretical value of $V_{g}$. A rectangular microwave input pulse of duration $T=29 \mathrm{~ns}$ and input power $P_{\text {in }}=10-700 \mathrm{~mW}$ was applied at the input antenna, which excited a two-dimensional spin wave packet with an almost cosinusoidal distribution of the wave amplitude along the $y$ axis, and a maximum amplitude near the center of the antenna. The initial sizes (at halfmaximum power) of the input wave packet along the $y$ and $z$ directions were determined by the temporal duration $T$ of the input microwave pulse $L_{z 0}=V_{g} T \cong 1.2 \mathrm{~mm}$, and by the length $l_{a}$ of the input antenna $L_{y 0} \cong l_{a}=2.5 \mathrm{~mm}$.

The distributions of spin wave intensity, proportional to the squared amplitude $m^{2}$ of instantaneous microwave magnetization, created in the YIG film by the propagating wave packet, were measured by a newly developed space- and time-resolved Brillouin light scattering (BLS) technique, used in the forward scattering geometry (for details, see [15]). In this technique the number of photons, having their frequency shifted by the frequency of the BVMSW mode, is proportional to the wave intensity. The laser spot (diameter $50 \mu \mathrm{m}$ ) was scanned across the area of $6 \times 6 \mathrm{~mm}^{2}$ between the antennas in steps of $0.1 \mathrm{~mm}$ starting from a distance $z_{0}=1 \mathrm{~mm}$ from the input antenna, while the input spin wave packet was being launched continuously with a repetition frequency of $F=1 \mathrm{MHz}$. At each point, the laser spot was held for $5 \mathrm{~s}$ to measure the complete time response of the local variable magnetization $\left.m^{2}(t)\right|_{(y, z)}(25<t<500 \mathrm{~ns})$ caused by the propagating spin wave pulse. To collect these time response data, electronic pulses caused by the detected photons were sorted by a fast (time resolution $1.8 \mathrm{~ns}$ ), multichannel analyzer according to their delay time with respect to the launch of the input microwave pulse. From these time-resolved data, collected at each spatial point, the two-dimensional distributions of the propagating spin wave packet intensity, corresponding to each delay time, were reconstructed. In our BLS experiment we were able to determine directly the local absolute value of the magnetization precession angle $\chi$, corresponding to a given value of the input power, by analyzing the ratio between the inelastically scattered light intensity and the incident light intensity, based on the relations given in Ref. [16]. The experimental details will be published elsewhere [15].

The experimentally measured two-dimensional distributions of the BVMSW intensity in a propagating wave packet obtained for $P_{\text {in }}=460 \mathrm{~mW}$, and corresponding to five different delay times, are shown in Fig. 1. The cross sections of the wave packets at the level of half-maximum are shown in the lower part of Fig. 1. The position of the 


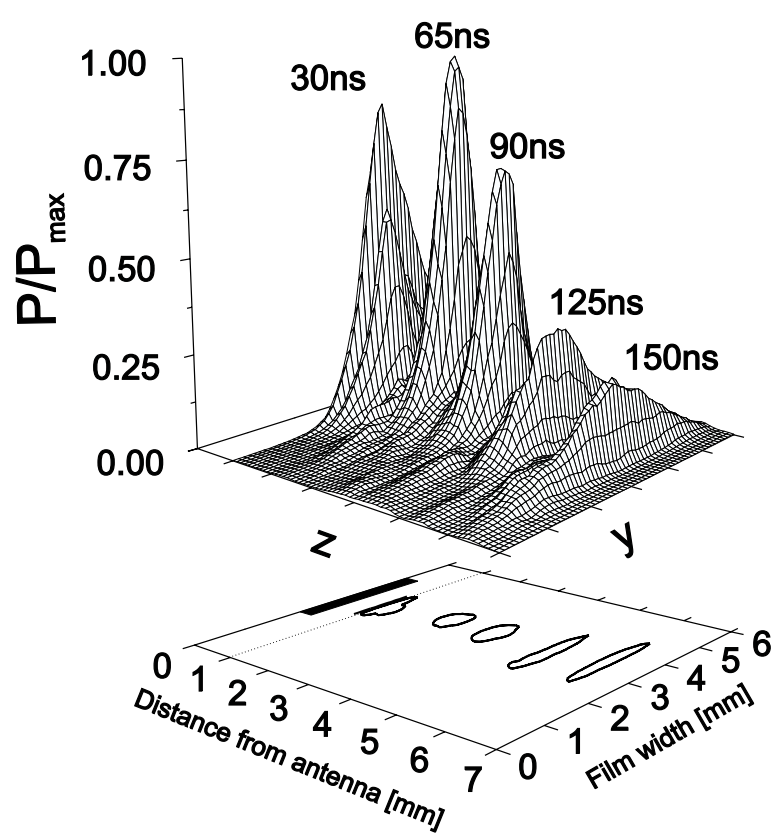

FIG. 1. Two-dimensional $(y, z)$ distributions of normalized intensity in propagating dipolar spin wave packets, corresponding to five different values of the propagation (delay) time as indicated in the figure. The distributions were experimentally measured by space- and time-resolved BLS technique for $T=$ $29 \mathrm{~ns}$ and $P_{\text {in }}=460 \mathrm{~mW}$. The cross sections of the propagating wave packets taken at half-maximum power are shown on the $(y, z)$ plane below.

input antenna is also shown at $z=0$. The data clearly demonstrate the existence of spatiotemporal self-focusing of the propagating wave packet with the focal point situated near $z=2-2.5 \mathrm{~mm}(t=50-60 \mathrm{~ns})$, where the peak amplitude of the wave packet has a maximum, and the packet width along the $y$ axis shows a minimum. This effect is further illustrated in Figs. $2 a$ and $2 b$, where the experimentally measured in-plane sizes $L_{z}$ and $L_{y}$ of the BVMSW packet cross sections (at half-maximum power) are shown as a function of the propagation time $t$ for the same input power $P_{\text {in }}=460 \mathrm{~mW}$.

For $t<40 \mathrm{~ns}$, the BVMSW packet, generated by the rf field of the antenna, is entering the region of the film accessible by BLS. Therefore, the visible size of the wave packet is linearly increasing with time. For $t=40-45 \mathrm{~ns}$, a rapid collapselike self-focusing of the packet is observed along both in-plane directions. Subsequently the collapse is stabilized by dissipation, and in the time interval $50<t<100 \mathrm{~ns}$ both in-plane sizes of the propagating packet are almost constant; i.e., a quasistable spin wave bullet is propagating in the film. For $t>100 \mathrm{~ns}$, the transverse size $L_{y}$ of the packet starts to increase rapidly due to the influence of diffraction. The dependence of the normalized peak power $P_{\text {peak }} / P_{\text {peak }}^{\max }$ of the wave packet on the propagation time for the input power $P_{\text {in }}=460 \mathrm{~mW}$ is shown in Fig. 2c. The focal point at $t=60 \mathrm{~ns}$ is clearly defined, and it corresponds to the minimum transverse size $L_{y}$ of the packet.

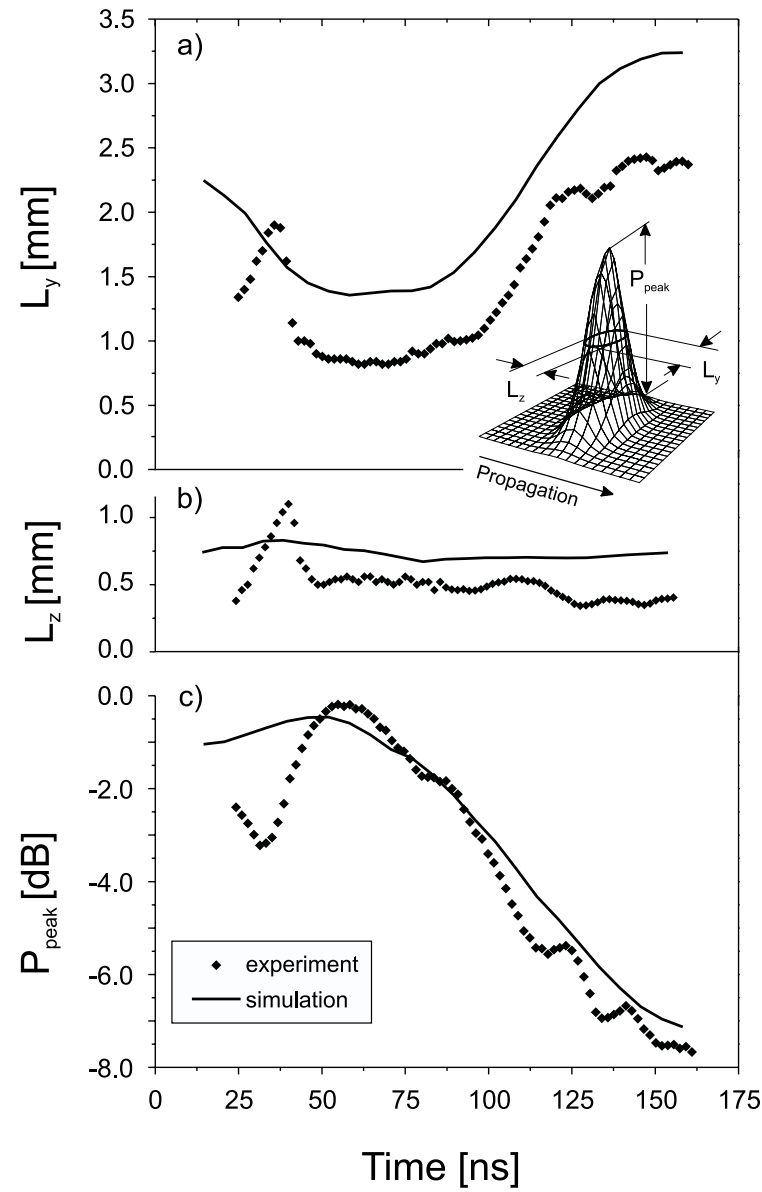

FIG. 2. Widths $L_{y}$ (a) and $L_{z}$ (b) at half-maximum power, and normalized peak power (c) of the propagating spin wave packet shown as functions of the propagation (delay) time $t$ for $T=29 \mathrm{~ns}$ and $P_{\text {in }}=460 \mathrm{~mW}$. Symbols: experiment; lines: numerical simulation.

The above-described spatiotemporal self-focusing effect was observed for $P_{\text {in }}>100 \mathrm{~mW}$. For lower input powers, we observed a monotonous decay of the packet peak amplitude, and also a monotonous increase of both packet sizes $L_{y}$ and $L_{z}$ with the increase of propagation time. Figure 3 demonstrates that at the focal point both $L_{y}$ and $L_{z}$ decrease with the increase of the input peak power of the BVMSW packet, i.e., that the effect of self-focusing and spin wave bullet formation is strongly nonlinear.

The above-discussed experimental data show that the initial wave packet, and the spin wave bullet formed from it, are both elliptic $\left(L_{z}<L_{y}\right)$. Thus, the analytic theory of the isotropic (cylindrical) collapse developed in Refs. [6,7] is not directly applicable to our case. Therefore, to model the observed effect of twodimensional anisotropic self-focusing of BVMSW packets in YIG films, we performed numerical simulations, based on Eq. (1) with the above-calculated parameters and elliptic initial wave packets, similar in shape to those used in the experiment.

For a direct comparison of the numerical results with the experimental data, we assumed that the initial amplitude 


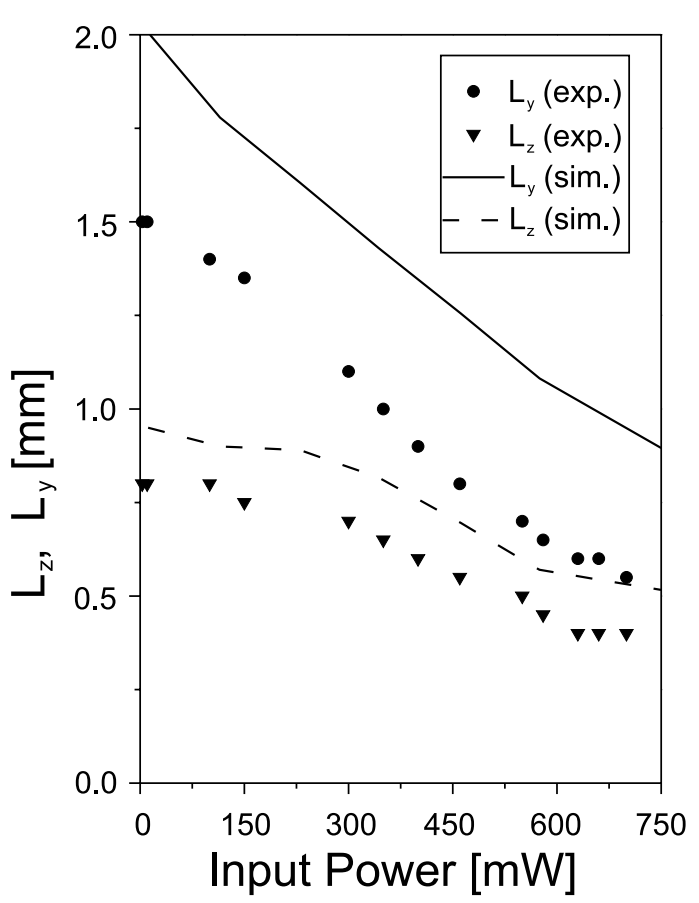

FIG. 3. Widths $L_{y}$ and $L_{z}$ of the dipolar spin wave packet at half-maximum power measured at the focal point (point of the maximum peak power) shown as functions of the input power $P_{\text {in }}$. Symbols: experiment; lines: numerical simulation.

$U_{0}$ corresponding to the input power $P_{\text {in }}=460 \mathrm{~mW}$ was $U_{0}=4.4 \times 10^{-2}$ in accordance with the experimentally measured value of the precession angle $\chi$.

The results of the numerical calculations are shown in Figs. 2 and 3 by solid lines. They are in good qualitative agreement with the experimental data, and the effect of two-dimensional spatiotemporal self-focusing is clearly seen in the numerical data. Although all salient features of the experiment are reproduced in the simulation, there is a discrepancy between the experimental and numerical values of the spin wave packet widths $L_{y}$ and $L_{z}$. This discrepancy can be related to the fact that our input spin wave packet was not exactly spectrally narrow in the wave number space $\left(\Delta k_{z}=\pi / L_{z 0}\right.$ was of the same order of magnitude as the carrier wave number $k_{z 0}=50 \mathrm{~cm}^{-1}$ ), and, therefore, the model (1) describes the evolution of the BVMSW packet only qualitatively. The systematically larger values of $L_{y}$ can also be caused by a too largely chosen initial pulse width $L_{y 0} \cong l_{a}$. Unfortunately $L_{y 0}$ is not directly accessible in the experiment, since the antenna region $(z<1 \mathrm{~mm})$ is not transparent for the laser light.

In conclusion, we have reported the first experimental observation of two-dimensional self-focusing of nonlinear dipolar spin waves in magnetic films, and formation of highly localized quasistable two-dimensional packets of spin waves - spin wave bullets. The stabilization of selffocusing and collapse in magnetic films happens due to the influence of dissipation that plays here a much more important role than for light pulses in optical fibers. This interpretation of the observed effect is supported by the numerical simulation based on the dissipative NLS equation.

This work has been supported by the Deutsche Forschungsgemeinschaft, by the National Science Foundation (Grant No. DMR-9701640), and by the Oakland University Foundation. The authors are grateful to B. Kalinikos and Yu. Kivshar for many helpful discussions. The YIG samples were kindly supplied by B. Kalinikos.

*To whom correspondence should be addressed.

[1] J. J. Rasmussen and K. Rypdal, Phys. Scr. 33, 481 (1986).

[2] P. L. Kelly, Phys. Rev. Lett. 15, 1005 (1965).

[3] S. A. Akhmanov, R. V. Khokhlov, and A.P. Sukhorukov, in Laser Handbook, edited by F. T. Arecchi and E. O. Schulz-Du Bois (North-Holland, Amsterdam, 1972).

[4] A. Y. Wong and P. Y. Cheung, Phys. Rev. Lett. 52, 1222 (1984).

[5] Y. Silberberg, Opt. Lett. 15, 1282 (1990).

[6] M. V. Goldman, K. Rypdal, and B. Hafizi, Phys. Fluids 23, 945 (1980).

[7] M. Desaix, D. Anderson, and M. Lisak, J. Opt. Soc. Am. B 8, 2082 (1991).

[8] A. N. Slavin, Phys. Rev. Lett. 77, 4644 (1996).

[9] A. N. Slavin, B. A. Kalinikos, and N. G. Kovshikov, in Nonlinear Phenomena and Chaos in Magnetic Materials, edited by P. E. Wigen (World Scientific, Singapore, 1994), Chap. 9.

[10] M. J. Lighthill, J. Inst. Math. Appl. 1, 269 (1965).

[11] O. von Geisau, U. Netzelmann, S. M. Rezende, and J. Pelzl, IEEE Trans. Magn. 26, 1471 (1990).

[12] M. Chen, M. A. Tsankov, J. M. Nash, and C. E. Patton, Phys. Rev. B 49, 12773 (1994).

[13] J. W. Boyle, S. A. Nikitov, A. D. Boardman, J. G. Booth, and K. Booth, Phys. Rev. B 53, 12173 (1996).

[14] M. Bauer, C. Mathieu, S. O. Demokritov, B. Hillebrands, P. A. Kolodin, S. Sure, H. Dötsch, V. Grimalsky, Yu. Rapoport, and A. N. Slavin, Phys. Rev. B 56, R8483 (1997).

[15] M. Bauer, O. Büttner, S. O. Demokritov, and B. Hillebrands, "Time- and Space-resolved Brillouin-LightScattering," Rev. Sci. Instrum. (to be published).

[16] H. L. Hu and F. R. Morgenthaler, Appl. Phys. Lett. 18, 307 (1971); W. Wettling, M. G. Cottam, and J. R. Sandercock, J. Phys. C 8, 211 (1975). 